\title{
Total collapse of the lung in aspergillosis
}

\author{
R. H. ELLIS \\ From the Gloucestershire Royal Hospital, Gloucester
}

Pulmonary aspergillosis can be divided conveniently into two main types, allergic and saprophytic (Hinson, Moon, and Plummer, 1952). Obstruction of the bronchi by mycelium and eosinophilic inflammatory exudate not uncommonly leads to segmental or lobar collapse, as was again pointed out by Hinson et al. (1952), and more recently by Villar, Pimentel, and Freitas E Costa (1962), Golberg (1962), and Wahner, Hepper, Andersen, and Weed (1963). I have found no reference in the literature to total lung collapse, so two examples of this serious complication of the disease, which have occurred during the past three years, are described below.

\section{CASE HISTORIES}

CASE 1 This housewife was a city-dweller aged 66, who had had periodic bronchitis for many years. In 1955 she first had asthmatic attacks, and in 1960 she was referred to the chest clinic with bronchitis and mild bronchospasm, and a chest radiograph showed early emphysema. Three months later she was admitted to the Gloucestershire Royal Hospital with severe dyspnoea and cyanosis. Her film showed collapse of the greater part of the left lung with infiltration in the posterior segment of the right upper lobe (Fig. 1). Re-expansion was achieved by bronchoscopic suction followed by intensive postural drainage and percussion. The bronchial aspirate was mucoid and grew Aspergillus fumigatus, which was later grown also from two specimens of sputum. The patient was under treatment with prednisone when discharged.

In 1961 she was re-admitted with severe dyspnoea and cyanosis together with bronchospasm. Her chest radiograph showed a lesion of the middle basal segment of the right lower lobe (Fig. 2). She deteriorated during the fourth week in hospital when a film showed the right lung-field to be clear, but there was total collapse of the left lung (Fig. 3). At bronchoscopy there were strings of mucus in all the major bronchial orifices and in the left main bronchus, but the airway was not completely obstructed. $\mathrm{Re}$ expansion was again achieved by bronchoscopic suction followed by prolonged postural drainage and percussion (Fig. 4). Bronchial aspirate and sputum cultures were negative for aspergillus but contained eosinophils. A blood eosinophilia was present $(2,373 /$ c.mm.), prick skin tests were strongly positive fo $\overrightarrow{B O}$ aspergillus, and serum precipitins were present agains extracts of $\boldsymbol{A}$. fumigatus. She was treated with predni $=$ sone and desensitization to $A$. fumigatus, and she remains well apart from some periodic bronchitis and wheeze.

CASE 2 A farmer's wife aged 60 was referred to thछ chest clinic in 1955 after pneumonia, when a cheser radiograph showed a lesion of the posterior basat segment of the right lower lobe (Fig. 5). She had of white cell count of $10,850 / \mathrm{c} . \mathrm{mm}$. with eosinophilp $756 / \mathrm{c} . \mathrm{mm}$. Three months later she was well with \& normal chest radiograph. In 1956 the film remaine clear, though there was some cough and mucoier sputum.

In 1963 she was short of breath with copious muco3 purulent sputum. A chest radiograph showed como plete collapse of the left lung (Fig. 6) and there was a blood eosinophilia of $1,464 / \mathrm{c} . \mathrm{mm}$. Her sputuri contained eosinophils and grew $A$. fumigatus. A bronchoscopy there was much mucus in the left maie bronchus and overlying the major bronchial orifices. but the airway was not completely obstructed. The lung re-expanded (Fig. 7) after bronchoscopic suction followed by intensive postural drainage with percuse sion. The bronchial aspirate grew $A$. fumigatus, the prick skin test was strongly positive for aspergillus, an $\Phi$ serum precipitins were present against extracts of $A_{D}$ fumigatus. She remains well apart from some periodio bronchitis.

\section{DISCUSSION}

In both the patients described there was a histor of chest symptoms dating back several years. If the first patient this began with asthma five years before she was admitted to hospital, and for man years before this she had had periodic bronchiti without wheeze. It is interesting that before the first episode of collapse of the left lung shg developed an infiltration in the right upper lobet A lesion of the right lower lobe was present af the second admission and just antedated the second episode of left-sided collapse.

In the second patient, the chest history begab with 'pneumonia' (also in the right lower lobe 


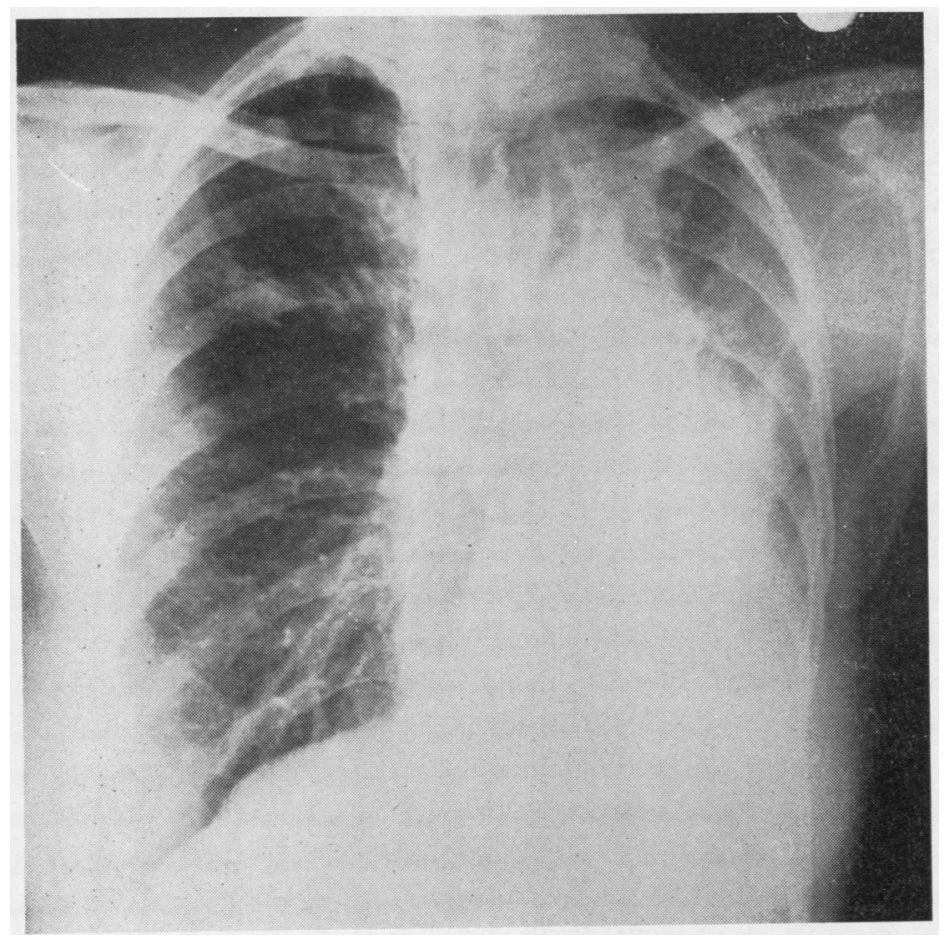

FIG. 1. Case 1, August 1960. Infiltration of the right upper lobe with collapse of the greater part of the left lung.

FIG. 2. Case 1, September 1961. Right upper lobe clear. Infiltration of the right lower lobe.

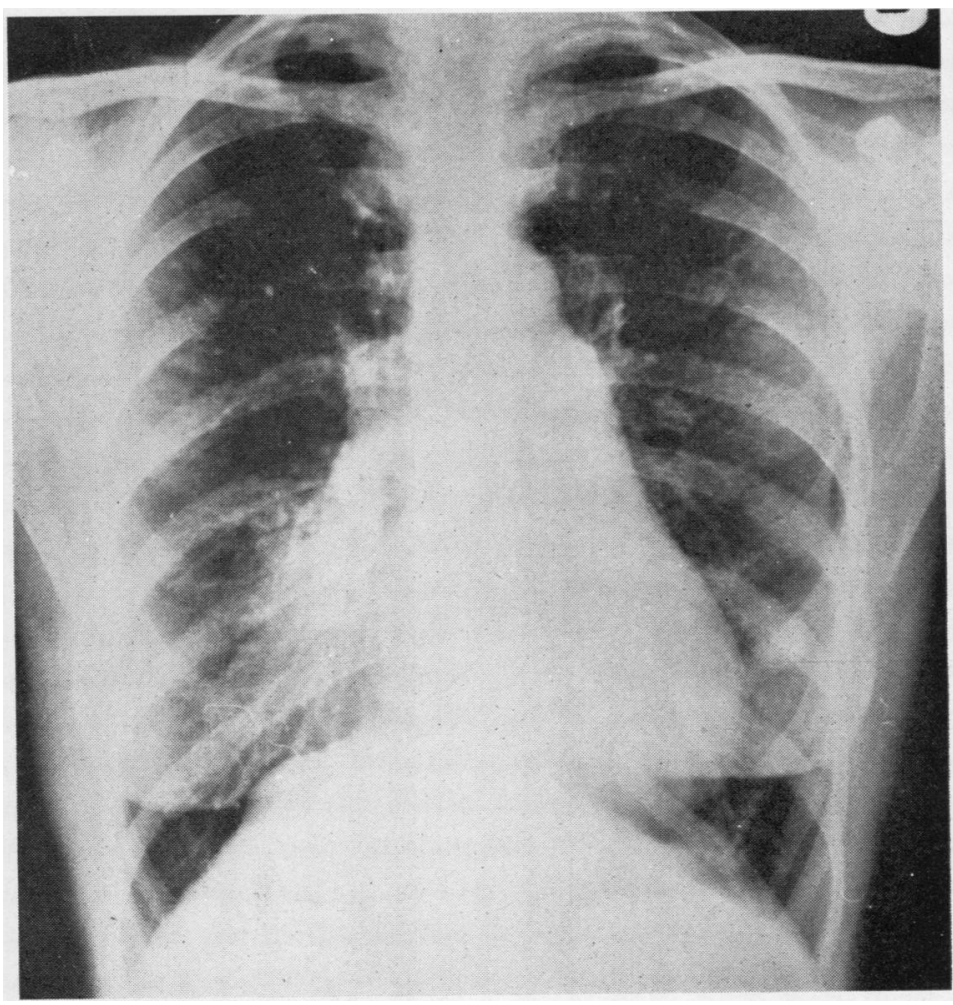




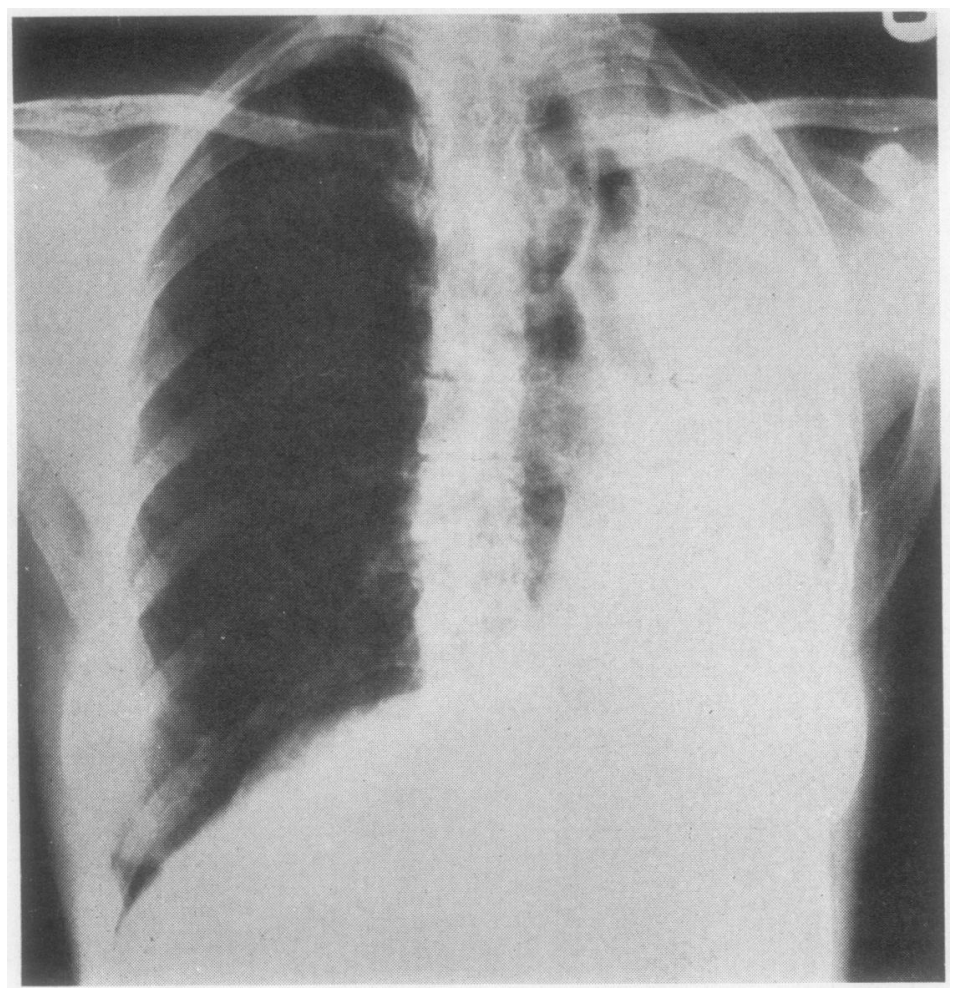

$$
\text { 검 }
$$

FIG. 3. Case 1, October 1961. Total collapse of the left lung.

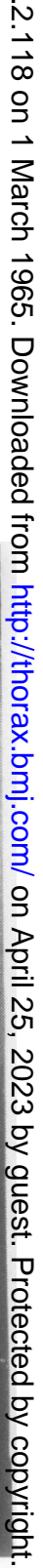

FIG. 4. Case 1, December 1961. Complete re-expansion of the left lung. The lung-fields are now clear.

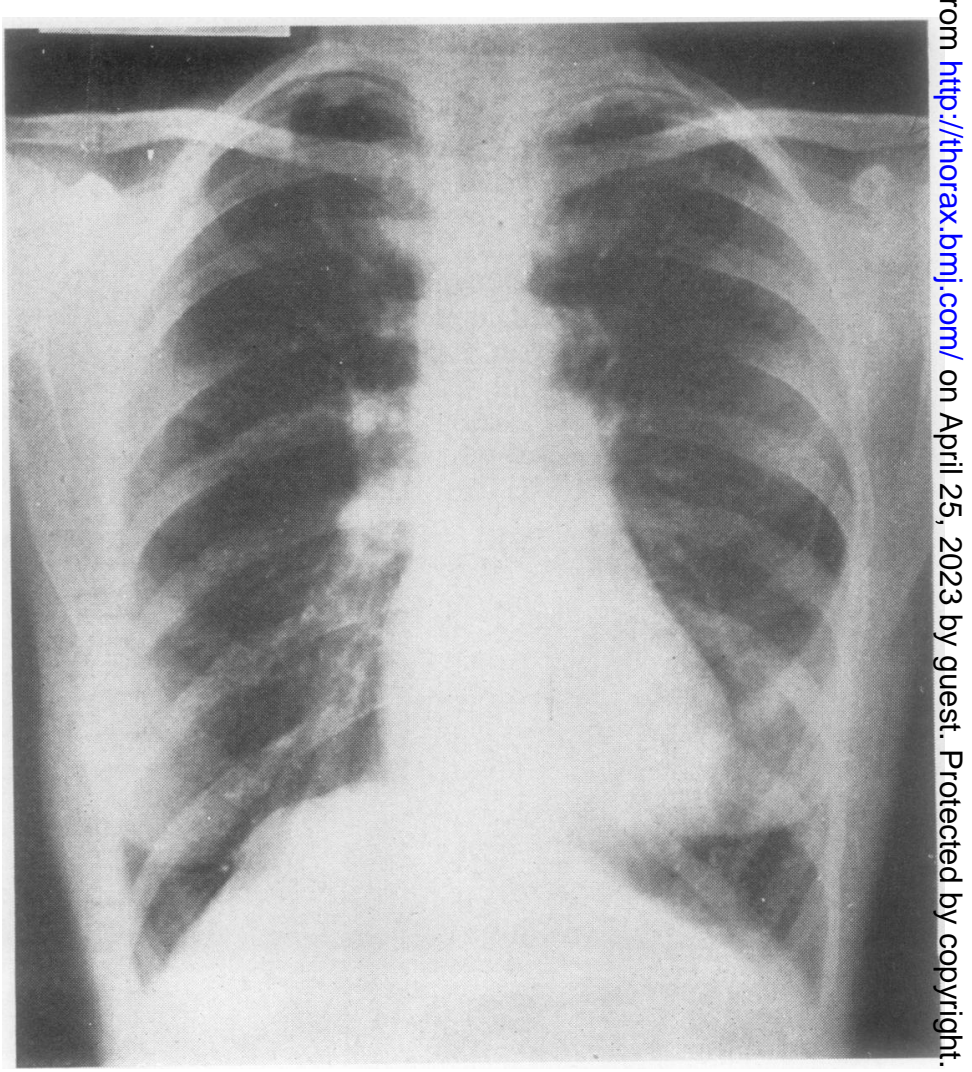




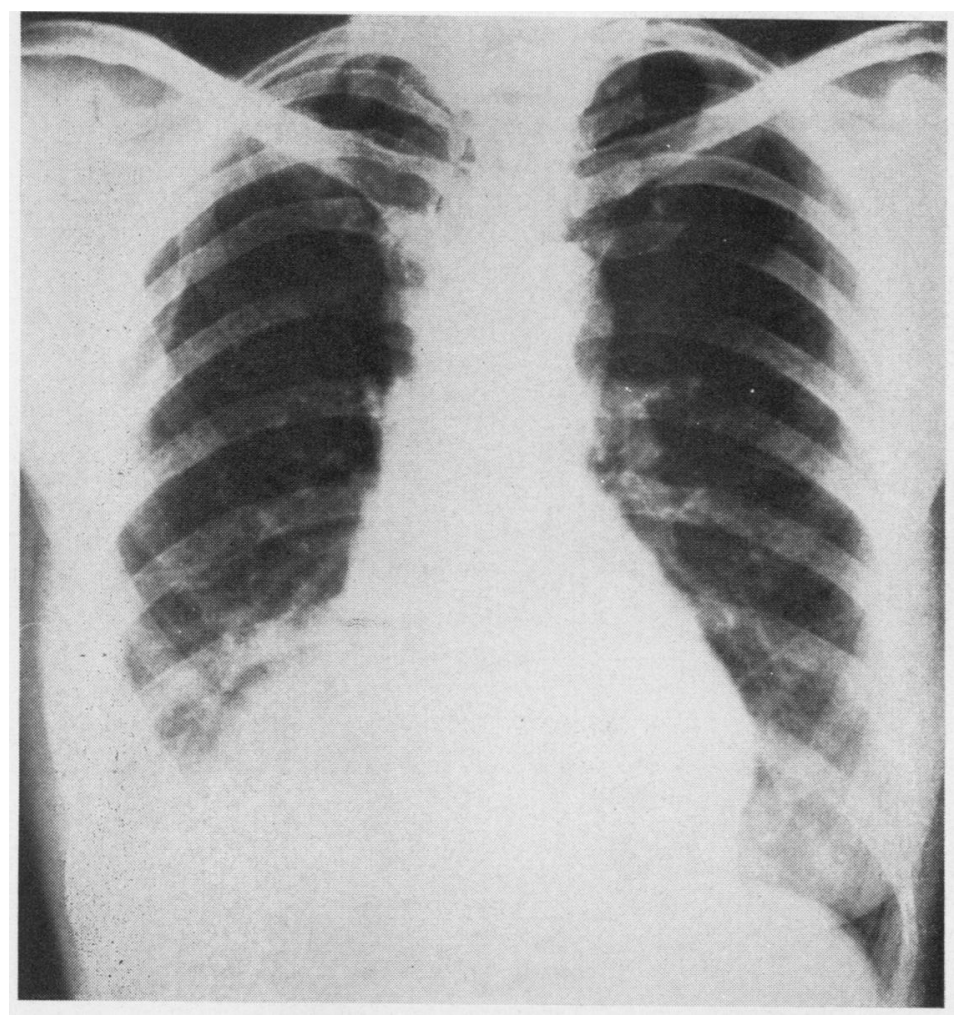

FIG. 5. Case 2, September 1955. Collapse of the posterior basal segment of the right lower lobe.

FIG. 6. Case 2, April 1963. Total collapse of the left lung. Small infiltration of the right upper lobe.

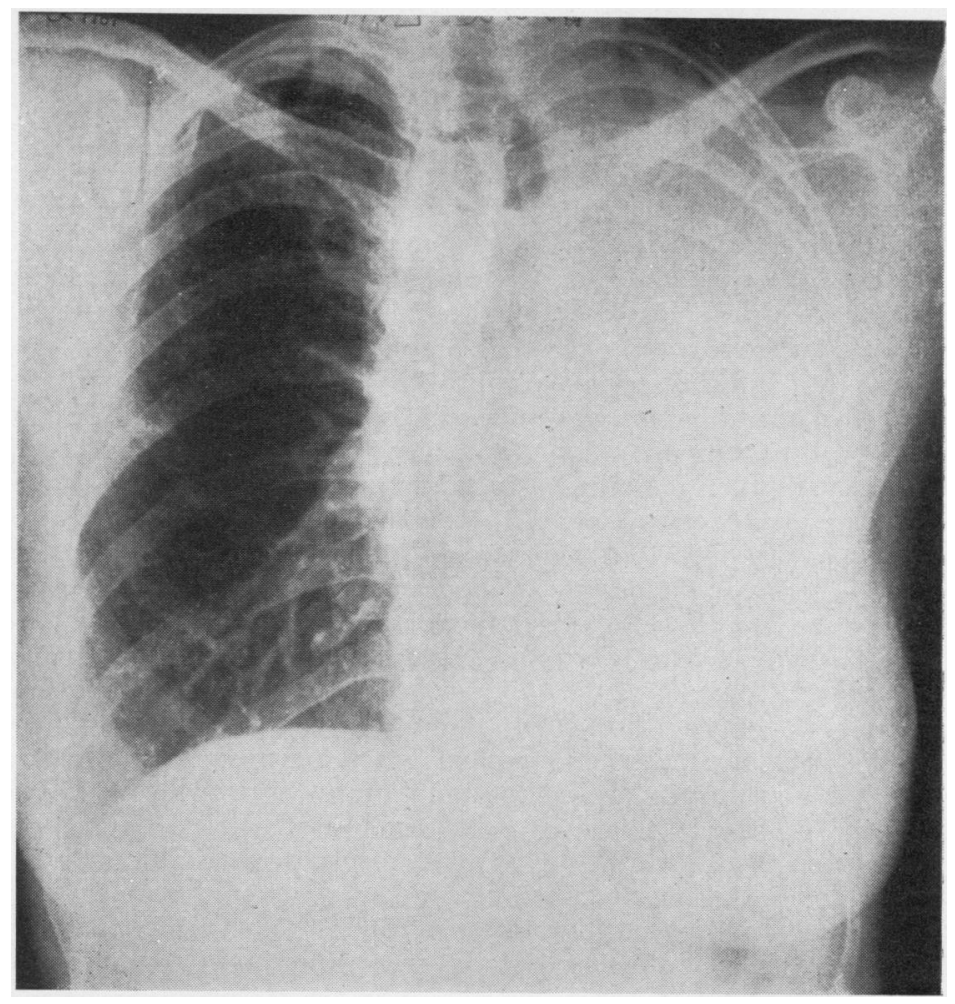




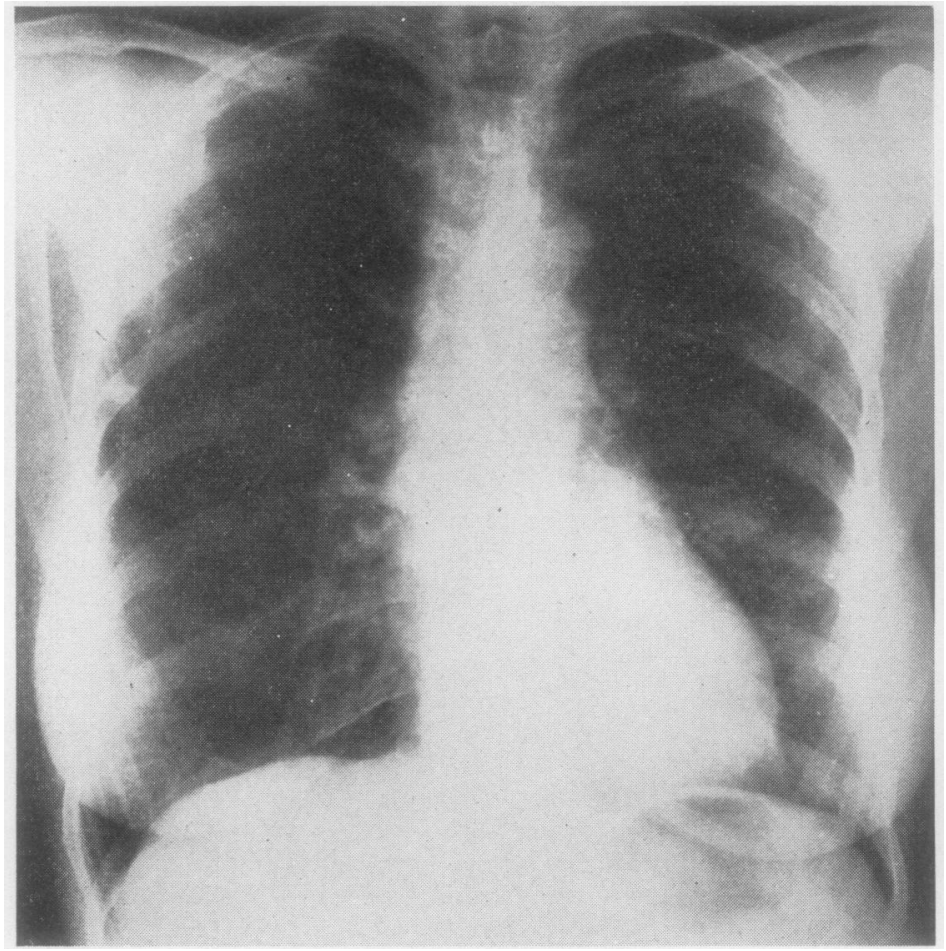

FIG. 7. Case 2, June 1963. Complete re-expansion of the left lung. Right upper lobe infiltration remains.

eight years before she was admitted to hospital with collapse of the left lung, and the pneumonia was followed by cough and sputum without wheeze for two years. She was symptom-free for five years before she was admitted to hospital. Although the chest films showed complete collapse of the left lung, at bronchoscopy obstruction of the left main bronchus was not found.

In both patients the major bronchi were patent, and though they contained much stringy mucus, this was insufficient to block the airway. Neither patient gave a history of coughing up brown plugs, though both complained of an increase of sputum during the month before their lungs collapsed. In the first patient, who was asthmatic, the wheezing increased.

Cultures of the bronchial aspirate grew $A$. fumigatus from the second patient but not from the first, and though several sputum cultures were positive in both, the growth was never profuse. Prick skin tests gave florid positive reactions to aspergillus in both cases, accompanied by a blood eosinophilia and by positive serum precipitin tests.

I thought that both these women were suffering from an allergic eosinophilic infiltration type of aspergillosis and that the lung collapse was duex to mucous obstruction of the bronchi. Since a all bronchoscopic examinations the left main. bronchus was not totally obstructed, probablye there was almost simultaneous obstruction of small branches after fragmentation and aspiration of a larger plug from the left main bronchus. This theory is supported by the fact that sucking out. mucus from the major bronchi did not result inf immediate re-aeration of the lung, and it required intensive postural drainage and percussion for three weeks before this was complete.

These patients resemble those described b Harvey, Blacket, and Read (1957), who conclude that the condition of mucous impaction of the bronchi resulted from a quantitative and qualita $=$ tive alteration of the mucus. Their patients were all asthmatics, however, and the episodes of collapse usually involved segments only. Further? although three of them had blood eosinophilia, n\& mention of aspergillus infection is made. It i interesting that six of their seven patients were found subsequently to have bronchiectasis. M 
two patients have not yet had bronchograms, though I think it unlikely that any severe bronchial damage has occurred in the involved lungs.

\section{SUMMARY}

Total collapse of a lung occurred in two patients with the allergic eosinophilic infiltration type of aspergillosis, with and without associated asthma. It was preceded by an increase of sputum and was associated with severe dyspnoea and cyanosis. It was probably caused by the simultaneous plugging of smaller bronchi by mucus aspirated from the main bronchi, which at bronchoscopy were found to be unobstructed.
Energetic and prolonged postural drainage and percussion, in addition to bronchoscopic suction, were necessary to achieve complete re-aeration of the lung.

\section{REFERENCES}

Golberg, B. (1962). Radiological appearances in pulmcnary aspergillosis. Clin. Radiol., 13, 106.

Harvey, C., Blacket, R. B., and Read, J. (1957). Mucoid impaction of the bronchi. Aust. Ann. Med., 6, 16.

Hinson, K. F. W., Moon, A. J., and Plummer, N. S. (1952). Brenchopulmonary aspergillosis. - A review ard a report of 8 new cases. Thorax, 7, 317 .

Villar, T. G., Pimentel, J. Cortez, and Freitas É Costa, M. (1962). The tumour-like forms of aspergillcsis of the lung (pulmonary aspergilloma). Thorax, 17, 22.

Wahner, H. W. Hepper, N. G. G. Andersen, H. A., and Weed, L. A. (1963). Pulmonary aspergiliosis. Ann. intern. Med., 58, 472 . 